\title{
Molecular Simulations of Surface Forces and Film Rupture in Oil/Water/Surfactant Systems
}

\author{
Live Rekvig, ${ }^{*} \dagger$ Bjørn Hafskjold, ${ }^{\dagger}$ and Berend Smit \\ Department of Chemistry, Norwegian University of Science and Technology, \\ N-7491 Trondheim, Norway, and Van't Hoff Institute for Molecular Sciences, University \\ of Amsterdam, Nieuwe Achtergracht 166, 1018 WV Amsterdam, The Netherlands
}

Received July 30, 2004. In Final Form: October 5, 2004

\begin{abstract}
We use dissipative particle dynamics (DPD) and molecular models to simulate interacting oil/water/ surfactant interfaces. The system comprises sections of two emulsion droplets separated by a film. The film is in equilibrium with a continuous phase, in analogy with the surface force apparatus. This is achieved by combining DPD with a Monte Carlo scheme to simulate a $\mu V T$ ensemble. The setup enables the computation of surface forces as a function of the distance between the two interfaces, as well as the detection of film rupture. We studied monolayers of nonionic model surfactants at different densities and compared oil-water-oil and water-oil-water emulsion films. Between surfactant monolayers facing each other tails-on (water-oil-water films), we observed repulsive forces due to the steric interaction between overlapping hydrophobic tails. The repulsion increases with surfactant density. Conversely, no such repulsion is observed between surfactant monolayers facing each other heads-on. Instead, the film ruptures, the monolayers merge, and a channel forms between the two droplet phases. Film rupture can also be induced in the water-oil-water films by forcing the interfaces together. The separation at rupture increases for oil-water-oil films and decreases for water-oil-water films when the surfactant density increases. The results are in qualitative agreement with existing theories of emulsion stability in creams, in particular with the channel nucleation theory based on the natural curvature of surfactants.
\end{abstract}

\section{Introduction}

The rate at which two droplets coalesce is very important in many fields, from crude oil recovery to cosmetics and food production. ${ }^{1}$ A key challenge for the oil industry is to separate oil droplets from the water phase. Conversely, the food and pharmaceutical industries usually need to prevent this separation. Although water-in-oil and oilin-water emulsions are thermodynamically unstable, surface-active molecules (surfactants and related molecules) can make them kinetically stable by preventing or slowing down the coalescence process.

Surfactants affect the coalescence rate in several ways: ${ }^{2}$ They self-assemble into a monolayer at the oil/water interface, thereby changing the film rupture probability. The adsorption also modifies the droplet-droplet interaction. Furthermore, surfactant aggregates can alter the hydrodynamic properties of the continuous phase and, with them, the droplet collision rate.

The relevant physical processes span a large range of time and length scales, making emulsion science a complex problem. In the hydrodynamic regime, droplet dynamics, flocculation, creaming, and sedimentation processes involve micrometer- to millimeter-sized drops and occur in seconds to months. On the other hand, film rupture involves reorientation of surfactant molecules, which takes place on nanosecond time scales.

Molecular simulation is a very attractive tool to study these events on the molecular scale. However, simulation at atomic resolution is computationally very expensive. We chose here a coarse-grained approach: dissipative

* Corresponding author. E-mail: live.rekvig@phys.chem.ntnu.no.

Norwegian University of Science and Technology.

$\pitchfork$ University of Amsterdam.

(1) Sjöblom, J.Emulsions and Emulsion Stability; Dekker: New York, 996

(2) Rosen, M. J. Surfactants and interfacial phenomena, 2nd ed.; Wiley: New York, 1989. particle dynamics (DPD) simulations of oil, water, and bead-spring surfactant models. Previously we used this technique to study the effect of surfactant structure on interfacial properties of oil/water/surfactant monolayers. ${ }^{3-6}$ This showed that these models reproduce experimental trends for interfacial tension and bending rigidity. In this paper we move on to interacting interfaces, to determine the effect of surfactant structure on droplet-droplet interactions and film rupture.

In a typical macroemulsion the droplet size is $1 \mu \mathrm{m}$ to $1 \mathrm{~mm}$. Due to limitations in computer speed and memory, droplets of colloidal sizes cannot be simulated with molecular detail. One alternative is to study smaller droplets, that are a few nanometers in radius and contain only a few hundred molecules. ${ }^{7,8}$ The unrealistically high curvature imposed by the restriction in droplet size may however affect the interfacial mechanisms.

Here this problem is solved by "zooming in" on the interface between two colloidal droplets; see Figure 1. On the molecular scale, the interface and surface chemistry come into focus. At this resolution, the emulsion interfaces are, on average, essentially flat in the sense that the droplet radius is much larger than the molecular scale. We simulate a rectangular box with an oil phase on each side, a water film in the middle (or vice versa), and surfactants at the two interfaces. Thus, computer power is focused on the interfacial region, omitting the large fraction of molecules that make up the bulk interior of the droplets and the reservoir of the continuous phase. This

(3) Rekvig, L.; Kranenburg, M.; Hafskjold, B.; Smit, B. Europhys. Lett. 2003, 63, 902-907.

(4) Rekvig, L.; Kranenburg, M.; Vreede, J.; Hafskjold, B.; Smit, B. Langmuir 2003, 19, 8195-8205.

(5) Rekvig, L.; Hafskjold, B.; Smit, B. J. Chem. Phys. 2004, 120, 48974905.

(6) Rekvig, L.; Hafskjold, B.; Smit, B. Phys. Rev. Lett. 2004, $92,116101$.

(7) Koplik, J.; Banavar, J. R. Science 1992, 257, 1664-1666.

(8) Murad, S.; Law, C. K. Mol. Phys. 1999, 96, 81-85. 


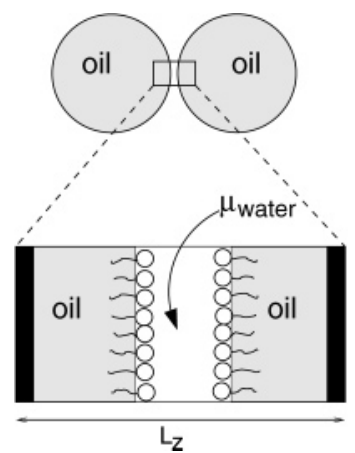

Figure 1. The surface of an emulsion droplet is, on average, essentially flat on a molecular scale. We simulate parts of two oil droplets separated by a water film and surfactants. The droplet separation is varied by adjusting the box length. To ensure equilibrium between the film and the continuous phase, the water chemical potential is fixed to that of bulk water.

reservoir is modeled implicitly by imposing an equilibrium between the film and a bulk reservoir. By doing this we obtain realistic force-distance curves as well as the critical film thickness at rupture. Hydrodynamic effects of the continuous phase, such as film draining, are left out. The system thus resembles creamed or sedimented layers, where film rupture is the rate-determining factor for stability.

A number of different forces may act between two colloidal particles: ${ }^{9}$ The Derjaguin-Landau-VerweyOverbeek (DLVO) forces include van der Waals and electrostatic interactions. Non-DLVO forces include steric interactions between chain molecules adsorbed at each surface. Packing effects in the solvent can cause oscillatory forces between rigid interfaces. Similarly, layering of micelles can cause oscillations on a larger scale. Finally, thermal fluctuations cause undulation and protrusion forces between liquid interfaces.

These forces can be measured using either hydrostatic or osmotic pressure techniques or the surface force apparatus. ${ }^{9}$ Simulations that, like ours, mimic the surface force apparatus, use grand canonical simulations to ensure equilibrium between the film and reservoir. This technique has been used extensively to study packing effects in solvents ${ }^{10}$ and recently also polymer brushes. ${ }^{11}$ In contrast, Nilsson et al. studied electrostatic and protrusion forces between amphiphilic surfaces separated by a continuous medium. ${ }^{12-14}$

With the present model we focus on steric and entropic forces. We use nonionic surfactants and liquid surfaces, which excludes electrostatic and oscillatory forces. The measured effective forces are similar to those observed for polymer brushes. ${ }^{11}$ The major difference is that the present interfaces are liquid (i.e., the surfactants are not anchored to the surface). Thermal undulations in the interface are thus preserved. Moreover, the "droplets" may coalesce, in the sense that the film ruptures, opposing surfactant monolayers merge, and the droplet interiors mix. We show here that in some cases the film ruptures prior to any steric interaction.

(9) Israelachvili, J. N. Intermolecular and Surface Forces, 2th ed.; Academic Press: London, New York, 1991.

(10) Gruhn, T.; Schoen, M. Mol. Phys. 1998, 94, 681-692.

(11) Goujon, F.; Malfreyt, P.; Tildesley, D. J. ChemPhysChem 2004 $5,457-464$

(12) Nilsson, U.; Jönsson, B.; Wennerström, H. Faraday Discuss. Chem. Soc. 1990, 90, 107-114.

(13) Nilsson, U.; Jönsson, B.; Wennerström, H.Adv. Colloid Interface Sci. 1992, 41, 179-195.

(14) Nilsson, U.; Jönsson, B.; Wennerström, H. J. Phys. Chem. 1993, $97,5654-5660$

\section{Model and Simulations}

2.1. Dissipative Particle Dynamics. In $D P D,{ }^{15-17}$ conservative, random, and dissipative forces act between two particles $i$ and $j$ which are a distance $r_{i j}$ apart

$$
\mathbf{F}_{i j}=\mathbf{F}_{i j}{ }^{\mathrm{C}}\left(r_{i j}\right)+\mathbf{F}_{i j}{ }^{\mathrm{R}}\left(r_{i j}\right)+\mathbf{F}_{i j}{ }^{\mathrm{D}}\left(r_{i j}\right)
$$

where the forces are of the form

$$
\begin{gathered}
\mathbf{F}_{i j}{ }^{\mathrm{C}}=a_{i j} w^{\mathrm{C}}\left(r_{i j}\right) \hat{\mathbf{r}}_{i j} \\
\mathbf{F}_{i j}{ }^{\mathrm{R}}=\sigma w^{\mathrm{R}}\left(r_{i j}\right) \theta_{i j} \hat{\mathbf{r}}_{i j} \\
\mathbf{F}_{i j}^{\mathrm{D}}=-\eta w^{\mathrm{D}}\left(r_{i j}\right)\left(\hat{\mathbf{r}}_{i j} \mathbf{v}_{i j}\right) \hat{\mathbf{r}}_{i j}
\end{gathered}
$$

Here, $\mathbf{v}_{i j}$ is the velocity difference for the two particles, $\hat{\mathbf{r}}_{i j}$ is the unit vector pointing from particle $j$ to particle $i$. $\theta_{i j}$ is a randomly fluctuating variable with Gaussian statistics, ${ }^{17} a_{i j}, \sigma$, and $\eta$ determine the amplitude of the conservative, random, and dissipative forces, respectively, while the $w$ functions are weight functions. To obey the fluctuation-dissipation theorem, we must have $w^{\mathrm{D}}=$ $\left(w^{\mathrm{R}}\right)^{2}$, and the system temperature will follow from the relation between $\sigma$ and $\eta: \sigma^{2} / \eta=2 k_{\mathrm{B}} T .{ }^{16}$ We use the commonly used weight functions ${ }^{16}$

$$
w^{\mathrm{C}}(r)=w^{\mathrm{R}}(r)=\left(w^{\mathrm{D}}(r)\right)^{1 / 2}=w(r)
$$

where

$$
w(r)= \begin{cases}1-\frac{r}{r_{\mathrm{c}}} & \text { for } r<r_{\mathrm{c}} \\ 0 & \text { for } r \geq r_{\mathrm{c}}\end{cases}
$$

Throughout this paper we use reduced units. The units of length, mass, and energy are the cutoff radius $r_{\mathrm{c}}$, bead mass $m$, and $k_{\mathrm{B}}$ times the temperature of the thermostat, respectively. We define $k_{\mathrm{B}} T=1$ at room temperature. In these units, $\sigma=3.0$ and $\eta=4.5$.

Integrating the equations of motion with a finite time step is nontrivial; the Verlet scheme, for example, cannot be applied to DPD in a straightforward manner because the dissipative force depends not only on the particle positions but also on their velocities. Several integration schemes have been proposed ${ }^{18}$ as well as a variation of the Anderson thermostat with some of the same features as the dissipative and random forces. ${ }^{19} \mathrm{We}$ have in this work opted for a new thermostat ${ }^{20}$ (the Peters thermostat) that is superior to those previously proposed for two reasons: First, it has been shown that all the time step dependence of static equilibrium quantities is due to the numeric integration of the conservative forces. ${ }^{20}$ Second, the effect of the thermostat reduces exactly to the effects of the dissipative and random forces in the limit of zero time step. The scheme involves first integrating the

(15) Hoogerbrugge, P. J.; Koelman, J. M. V. A. Europhys. Lett. 1992 $19,155-160$

(16) Español, P.; Warren, P. B. Europhys. Lett. 1995, 30, 191-196.

(17) Groot, R. D.; Warren, P. B. J. Chem. Phys. 1997, 107, 44234435

(18) Nikunen, P.; Karttunen, M.; Vattulainen, I. Comput. Phys. Commun. 2003, 153, 407-423.

(19) Lowe, C. Europhys. Lett. 1999, 47, 145-151.

(20) Peters, E. A. J. F. Europhys. Lett. 2004, 66, 311-317. 


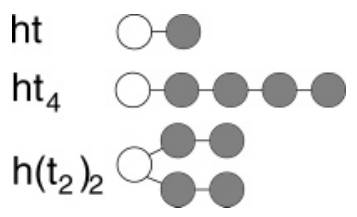

Figure 2. Some of the model surfactants investigated in this study and the nomenclature used. The white particles are hydrophilic beads (h) and the gray particles hydrophobic tails (t).

Table 1. Repulsion Parameters $a_{i j}{ }^{a}$

\begin{tabular}{cccc}
\hline & w & o & h \\
\hline $\mathrm{w}$ & 25 & 80 & 15 \\
$\mathrm{o}$ & 80 & 25 & 80 \\
$\mathrm{~h}$ & 15 & 80 & 35
\end{tabular}

${ }^{a}$ Key: $\mathrm{w}=$ water bead, $\mathrm{o}=$ oil or tail group, and $\mathrm{h}=$ head group.

conservative forces using, for example, the Verlet algorithm. Subsequently, the velocities are thermostated in a manner that rigorously maintains the Maxwell distribution. A more detailed discussion and comparison of the integration schemes is given in Appendix A.

2.2. Model. We use a coarse-grained approach where one DPD particle represents a group of atoms or a liquid volume. Water beads, oil beads, headgroups, and tail groups are denoted by w, o, h, and t, respectively. The tail beads are identical to the oil beads. Some of the model surfactants investigated are shown in Figure 2. A surfactant molecule consists of headgroups and tail groups connected by harmonic springs

$$
\mathbf{F}_{i j}{ }^{\text {Bond }}=-k_{\mathrm{s}}\left(r_{i j}-r_{0}\right) \hat{\mathbf{r}}_{i j}
$$

with $k_{\mathrm{S}}=100$ and $r_{0}=0.7$. Water and oil are represented by a single bead for simplicity. One tail bead typically represents a few $\mathrm{CH}_{2}$ groups. ${ }^{21-23}$ The repulsion parameters used are shown in Table 1 . These are taken from Groot, ${ }^{22}$ except that we used $a_{\mathrm{oo}}=25$ instead of 15 . This change has been made to obtain similar bulk densities in the oil and in the water phase (see also ref 4). The hydrophobic effect is mimicked with a high repulsion between hydrophilic and hydrophobic beads. Since the forces are short range, the model is suitable for nonionic surfactants.

2.3. Surface Forces. The system consists of a rectangular box with a bulk phase on each side, a film in the middle, and surfactants at the two interfaces (Figure 1). For now we assume two oil phases separated by a water film (in case of an oil film separating two water phases, simply swap oil and water in the following description). Our objective is to measure the effective forces between the interfaces as a function of the separation between them for various types and area densities of surfactants. The simulation box has a constant area $L_{x} \times L_{y}$. The box length $L_{z}$ is fixed in each simulation but is systematically decreased in order to vary the separation between the two interfaces. The system is periodic in $x$ and $y$ directions, but in the $z$ direction there are soft repulsive walls. The walls are included to ensure that all interactions between the two interfaces occur via the film.

When the system is in mechanical equilibrium, the forces between the interfaces will equal the forces exerted by the particles on the walls, and the force per area will equal the normal pressure in the system. Computing these

(21) Prinsen, P.; Warren, P. B.; Michels, M. A. J. Phys. Rev. Lett. 2002, 89, 148302.

(22) Groot, R. D. Langmuir 2000, 16, 7493-7502

(23) Groot, R. D.; Rabone, K. Biophys. J. 2001, 81, 725-736. forces in an NVT simulation does not give the desired insight because the normal pressure depends on the overall density of the system $\rho=N / V$, where $N=N_{\text {w }}+N_{\text {o }}+N_{\text {surf }}$, as well as on the composition. For example, a bulk system and a heterogeneous system with the same $N / V$ will have different $P_{\text {normal }}$, and the difference will depend on the size of the bulk phases compared to the interfacial area and not only the film thickness. Alternatively, imposing $P_{\text {normal }}$ is only suitable for measuring monotonically repulsive forces and not both sides of an energy barrier. ${ }^{14}$

We therefore combined DPD with a Monte Carlo (MC) scheme to simulate a $\mu V T$ ensemble (details on this combination can be found in ref 24 and, e.g., ref 4). The box dimensions as well as the number of oil and surfactant molecules are fixed, while the number of water molecules is varied according to a fixed chemical potential. We used $\mu_{\text {water }}=\mu^{\text {res }}=13.27$, which corresponds to bulk water at $\rho=3.0$. This ensures that the water film is always in equilibrium with bulk water. As we systematically decrease $L_{z}, N_{\text {water }}$ and the film thickness will decrease until the film is in equilibrium with the bulk reference state.

The forces between the interfaces can then be interpreted in the following way: Imposing $\mu_{\text {water }}$ ensures that the film is always in equilibrium with bulk water at $\rho=$ 3.0 , which has a pressure of $P^{\text {res }}=23.65$. In the heterogeneous system, the tangential pressure varies through the box: In the vicinity of the walls it oscillates as a function of $z$ due to packing effects, and on the interfaces it decreases due to the tension. However, the normal pressure is constant through the system. Furthermore, any discrepancy from the bulk pressure at $\mu_{\text {water }}$ is due to the interfaces. $P_{\text {normal }}>P^{\text {res }}$ means effective repulsion between the two bulk phases (thought of as droplets) and $P_{\text {normal }}<P^{\text {res }}$ means effective attraction. By fixing the box length and the chemical potential of the film in this manner, one can simulate both sides of a possible energy barrier between the drops.

This method is similar to experiments and simulations of a confined liquid between two parallel plates. A surface force apparatus can be used to measure the force on the plates as a function of the separation between them. ${ }^{25,26}$ In simulations, the normal pressure is sampled as a function of the plate separation at an imposed chemical potential. ${ }^{10,11}$ In this study we extended this method by adding a bulk phase and surfactants between the walls and the film. Also, the distance of interest is that between the interfaces rather than that between the walls. Although the number of oil particles is the same in all simulations, the density in the oil phase may vary if the pressure changes with box length. In section 3.2 we will discuss how to compute the separation between the droplet interfaces.

2.4. Wall Potential. Without walls, i.e., with periodic boundary conditions also in the $z$ direction, it is possible that interfacial undulations may propagate across the periodic box. Preventing such interaction is the sole purpose of the walls. Since $\mu_{\text {water }}$ is imposed, the wall potential does not influence the equilibrium state far away from the walls and the form of the wall potential is not important. There are two practical issues to consider, however: First, one would like a wall potential that gives a minimum of packing effects close to the walls. Second, because $\mu_{\text {water }}$ is imposed with acceptance probability

(24) Willemsen, S. M.; Vlugt, T. J. H.; Hoefsloot, H. C. J.; Smit, B J. Comput. Phys. 1998, 147, 507-517.

(25) Israelachvili, J. N.; Tabor, D. Proc. R. Soc. London, Ser. A 1972, $331,19-38$.

(26) Israelachvili, J. N.; McGuiggan, P. M. Science 1988, 241, 795800 . 


$$
\begin{aligned}
& \operatorname{acc}\left(N_{\mathrm{w}} \rightarrow N_{\mathrm{w}}+1\right)= \\
& \min \left(1, \frac{V}{N_{\mathrm{w}}+1} \exp \left\{\left[\mu_{\mathrm{w}}-\Delta U\right] / k_{\mathrm{B}} T\right\}\right)
\end{aligned}
$$

one needs a well-defined volume $V$. If the wall potential is such that some of the volume of the box is inaccessible, the density in bulk (away from the packing effects) will differ in a system with and without walls.

We therefore chose a soft repulsive wall of the same form as the interparticle potential, with parameters to minimize the packing effects.

$$
\mathbf{F}_{i}^{\text {wall }}=a_{\text {wall }, i} w\left(\left|z_{\text {wall }, i}\right|\right) \hat{\mathbf{z}}_{\mathrm{wall}, i}
$$

where $z_{\text {wall }, i}=z_{\text {wall }}-z_{i}$ and $\hat{\mathbf{z}}_{\mathrm{wall}, i}=\left(z_{\text {wall }, i} /\left|z_{\text {wall }, i}\right|\right) \hat{\mathbf{z}}$. With this potential there is a finite density in all regions of the box. To prevent particles from crossing the box boundary in the $z$ direction, we added hard reflective walls:

$$
\begin{aligned}
& \text { if } z_{i}(t+\Delta t)<0.0 \text { then } z_{i}(t+\Delta t)=-z_{i}(t+\Delta t) \\
& \text { if } z_{i}(t+\Delta t)>L_{z} \text { then } z_{i}(t+\Delta t)=2 L_{z}-z_{i}(t+\Delta t)
\end{aligned}
$$

To investigate the influence of various walls on the system, we studied in detail a one-component system of dimensions $5 \times 5 \times L_{z}$ at $\mu=13.27$. Figure 3 shows that the system size and wall repulsion parameters do not affect the bulk densities. Figure 3 a shows the packing effects close to the walls. Comparing $a_{\text {wall }}=0,10,25$, and 40, it shows that $a_{\text {wall }}=25$ gives the smallest oscillations. We therefore chose $a_{\text {wall,w }}=a_{\text {wall,h }}=25$ and $a_{\text {wall,o }}=a_{\text {wall,t }}=80$ for water droplets separated by an oil film, and $a_{\text {wall,w }}=a_{\text {wall,h }}=80$ and $a_{\text {wall,o }}=a_{\text {wall,t }}=25$ for oil droplets separated by a water film.

Figure 4 shows density and pressure profiles in a bulk $\mu V T$ ensemble, with and without walls. The density and tangential pressure profiles oscillate close to the wall due to packing effects. The normal pressure, however, is constant through the system (we have not included the contribution of the wall forces to the pressure, it therefore differs within a distance $r_{\mathrm{c}}$ from the wall). At a distance of $2-3 r_{\mathrm{c}}$ away from the walls, there is no significant difference between the system with and without walls.

2.5. Simulation Details. The number of oil molecules was fixed at 3628 . The number of water molecules fluctuates since only its chemical potential is imposed, with an average determined by the box length. The number of surfactant molecules was fixed in each simulation but varied to simulate the effect of variations in monolayer density. Starting with box dimensions of $11 \times 11 \times 23$, $L_{z}$ was systematically decreased to model two approaching interfaces. In the density ranges applied here, there are
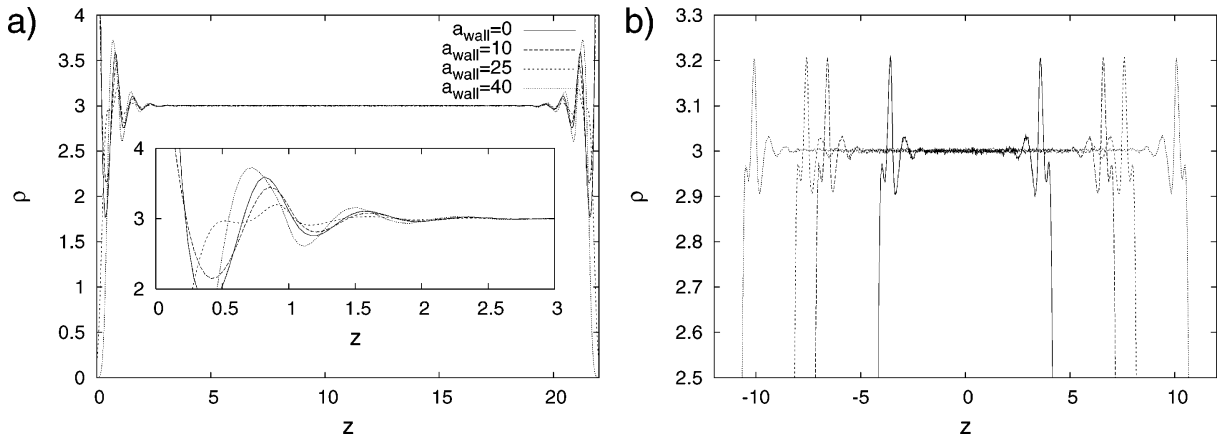

Figure 3. Density profiles for various wall repulsion parameters at $L_{z}=20$ (a) and for various system sizes at $a_{\mathrm{wall}}=25$ (b) for bulk water (or oil) at $\mu=13.27$. virtually no surfactants in the film phase, so the monolayer density remains constant during compression.

We performed both nonequilibrium simulations, in which the box length was decreased continuously, and equilibrium simulations. In the nonequilibrium simulations, the box length was continuously decreased at a speed low enough to allow the number of film molecules to adjust accordingly. This corresponds to "pushing" the two drops together, but slowly enough for the particles to diffuse out. In practice particles are added and removed uniformly in the box with probability according to their Boltzmann weight. A reduction in box length of $\left(2 \times 10^{-5}\right) r_{\mathrm{c}}$ per MC step was found to be sufficiently low to keep a constant pressure (Figure 5a) and densities indistinguishable from those obtained at even lower speed (Figure 5b). Nevertheless, the separation at rupture may depend on the speed of approach and on the system size. However, all simulations are at the same speed and system size and the qualitative differences observed are therefore caused by different surfactants.

Each MC step consists of an attempt to add/remove a water particle or a series of RAN $\times 200$ DPD steps with $\Delta t=0.03$, where $\mathrm{RAN}$ is a random number between 0 and 1. The probability of particle insertion/removal was set to 0.7 during the box decrease and 0.5 during equilibration and sampling. The pobability of trial insertion equals the probability of trial removal. For insertion, a random position is chosen uniformly in the box, and for removal, a water particle is chosen at random. Hence, the usual acceptance rules for the grand canonical Monte Carlo apply. ${ }^{33}$ In the nonequilibrium runs, configurations were saved at box length intervals of $0.5 r_{\mathrm{c}}$. These were used to sample equilibrium properties for $50000 \mathrm{MC}$ steps. The reference state was bulk water at $\rho=3.0$, for which $P^{\text {res }}$ $=23.65$ and $\mu^{\text {res }}=13.27$.

\section{Characterization of the System}

3.1. Detecting Film Rupture. The system can be thought of as a "zoom in" on two droplets separated by a film of the continuous phase. At this scale the surface of micrometer-sized droplets is practically planar on average. Droplets that have aggregated due to creaming or sedimentation may also be deformed, making the interface planar also on a mesoscopic scale. ${ }^{27}$

In real systems, coalescence occurs when the film ruptures, a channel between the two droplet phases is established, the channel radius expands, and the droplets' interiors mix. In the simulation system, the radius of the channel can only expand to a certain limit due to the periodic boundary conditions. This limit is determined by the box area. Moreover, the periodic boundary conditions cause subsequent changes in topology which do not have analogies in real systems but are relevant for a correct 

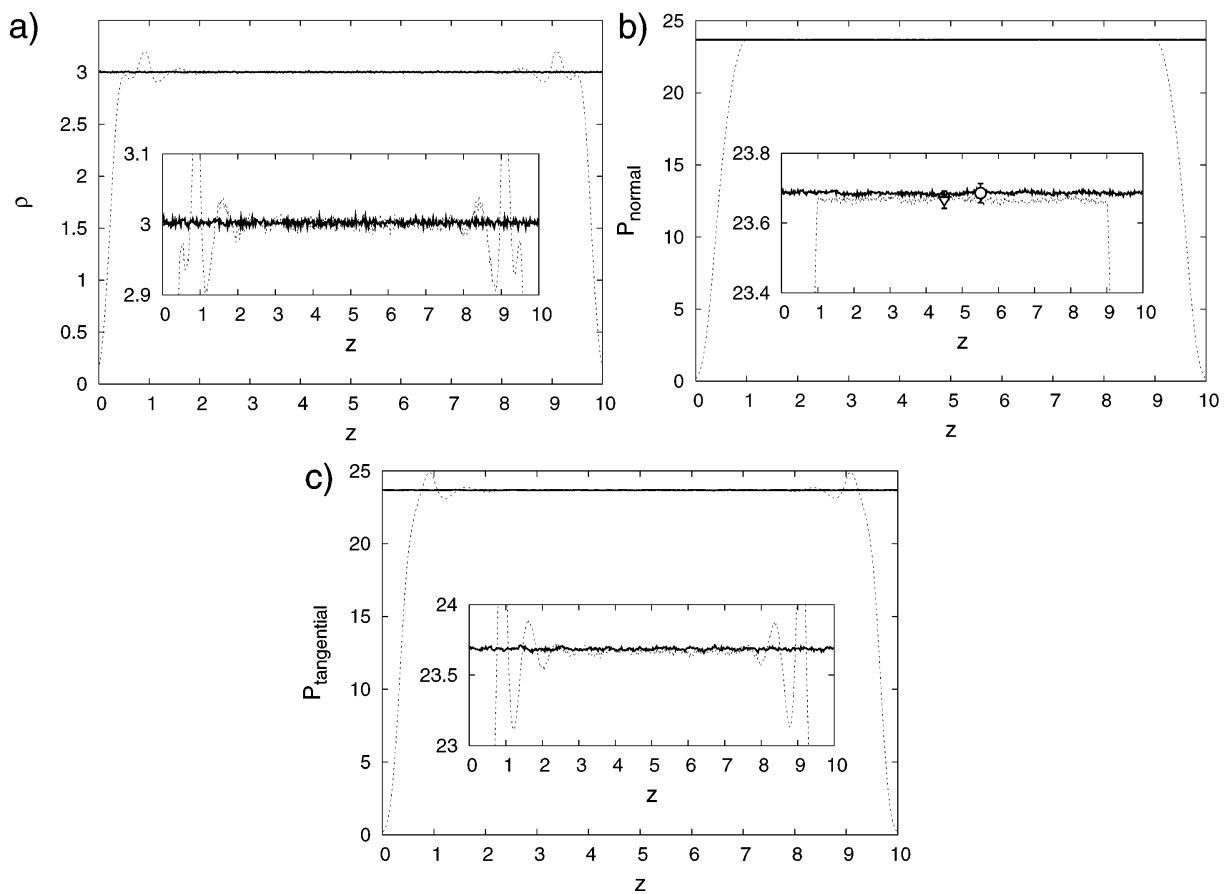

Figure 4. (a) Density, (b) normal pressure, and (c) tangential pressure in a bulk water (or oil) system at $\mu=13.27$ without walls (solid lines) and with walls with $a_{\text {wall }}=25$ (dashed lines). In the inset in (b) the points (triangle for system with walls, circle for system without walls) denote averages in time and in space in slabs more than $1 r_{\mathrm{c}}$ away from the walls. The error bars show three standard deviations based on independent time averages.
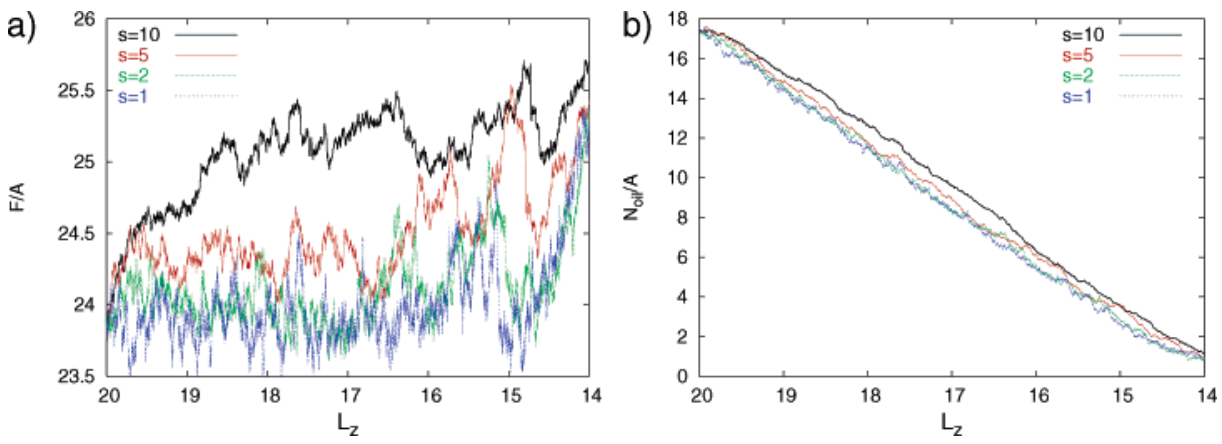

Figure 5. The system was compressed at a speed of $\left(s \times 10^{-5}\right) r_{\mathrm{c}}$ per MC step, with $s=10$ (black), $s=5$ (red), $s=2$ (green), and $s=1$ (blue). The surfactant was of type ht $t_{4}$ and the density $N_{\text {surf }} / A=0.5$. (a) Force on the walls per unit area as a function of box size. (b) Number of film molecules per unit area as a function of box size.

interpretation of the simulation results. In the following we assume a water film separating two oil phases and a high interfacial tension such that the global equilibrium state is that of minimum surface area.

From an equilibrium point of view, the film slab breaks into a droplet when the droplet area is lower than the corresponding slab area. If the film thickness is $l$ and the box area is $L^{2}$, the volume of the water phase is $L^{2} l$ and the area is $2 L^{2}$. The area of a sphere of the same volume is $2 L(\pi L l)^{1 / 2}$. This means that the sphere is preferred to a slab when $l<0.32 L$. However, in going from a film to a sphere, there are three topological transitions: film $\rightarrow$ channel $\rightarrow$ cylinder $\rightarrow$ sphere. Figure 6 shows these transitions seen from cuts along the $x-y$ planes at $z=$ $L_{z} / 2$ (top), and along the $x-z$ planes at $y=L_{y} / 2$ (bottom). To determine which topology the system has at a given time during the simulation, we define an alignment parameter $S_{\alpha}$

$$
S_{\alpha}=\left\langle 3 \cos ^{2} \theta_{\alpha}-1\right\rangle / 2
$$

where $\theta_{\alpha}$ is the angle between the bond that connects
Table 2. Typical Values of $S_{\alpha}$ in the Four Topologies Shown in Figure 6

\begin{tabular}{clll}
\hline film & channel & cylinder & sphere \\
\hline$S_{x}<0$ & $S_{x}<0$ & $S_{x}>0$ & $S_{x}=0$ \\
$S_{y}=S_{x}$ & $S_{y}=S_{x}$ & $S_{y}<0$ & $S_{y}=0$ \\
$S_{z}>0$ & $S_{z}>0$ & $S_{z}=S_{x}$ & $S_{z}=0$
\end{tabular}

hydrophilic and hydrophobic surfactant beads and the normal in direction $\alpha$ as defined by the simulation box. The brackets denote the average over all such bonds. This parameter is thus a measure of the alignment of the surfactants in a certain direction. For a surfactant film in the $x-y$ plane, $S_{x}=S_{y}=-0.5$ and $S_{z}=1$ if the bonds were perfectly aligned along the $z$ direction. Another limiting case is a spherical droplet; then $S_{x}=S_{y}=S_{z}=$ 0 . Table 2 shows characteristic values for the four topologies shown in Figure 6. Note that $S_{x}+S_{y}+S_{z} \equiv$ 0 .

From a nonequilibrium point of view, the separation at which the transitions occur depends on other factors than

(27) Ivanov, I. B.; Danov, K. D.; Kralchevsky, P. A. Colloids Surf., A 1999, 152, 161-182. 


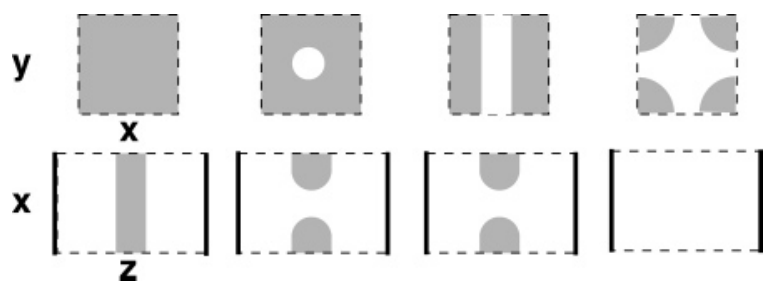

Figure 6. Cartoon showing the topological states. From left to right, the system has the topology of a film, a film with a hole (the channel state), a cylinder, and a sphere (due to the periodic boundary conditions). The cuts are along the $x-y$ planes at $z$ $=L_{z} / 2$ (top), and along the $x-z$ planes at $y=L_{y} / 2$ (bottom). The film phase is in gray. Dashed lines indicate periodic boundary conditions, full lines indicate walls.

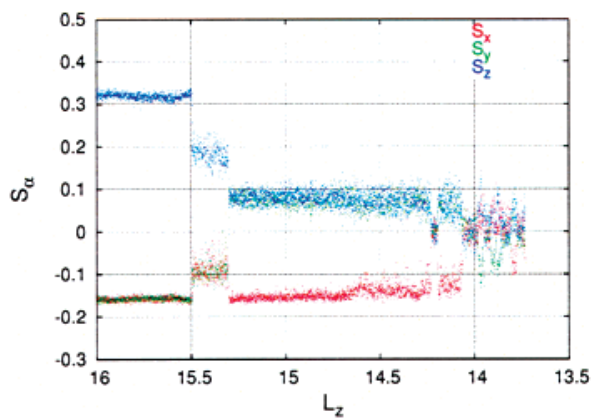

Figure 7. Changes in the alignment parameters as the box length is systematically decreased. The film, channel, cylinder, and sphere topologies can be identified. Each dot represents an average over 1000 DPD steps. The surfactant is $h_{2} t_{2}$ with branched tail and $N_{\text {surf }} / A=0.8$.

simply minimizing the surface area. Formation of a channel is the first step both in real droplet-droplet coalescence and for the subsequent transitions outlined in Figure 6. Without surfactants, this event occurs spontaneously following the initial contact between the drops. With surfactants present, formation of a channel requires reorientation of the surfactant molecules near the point of rupture. This can cost considerable energy, depending on the elastic properties of the monolayer. Kabalnov and Wennerström ${ }^{28}$ derived an expression for this energy barrier. The channel free energy has four terms: A negative term due to reduction in the planar area, a positive term due to the area of the channel, and a bending term proportional to the bending modulus and with sign depending on whether the bending is toward or against the natural monolayer curvature. The fourth term is a constant due to the topology change and determined by the saddle splay modulus. In this context, film rupture in aggregated droplets is a thermally activated event. Such events rarely occur spontaneously during the short time covered by a simulation. Therefore, we force instead the monolayers slowly together and monitor the separation at which the film ruptures. The separation at rupture gives an idea of the barrier.

Figure 7 shows how the alignment parameters change when two droplets approach each other. Initially, the surfactant films are in the $x-y$ plane with a positive $S_{z}$ and negative and equal $S_{x}$ and $S_{y}$. At a certain separation, the films come in contact and a channel is formed between them. The radius of the channel expands, which is observed as a rapid decrease in $S_{z}$ and increase in $S_{x}$ and $S_{y}$. The second plateau is a channel state with a radius determined by the box area. In an infinite system, the channel radius will expand until the original two droplets have merged to one single drop. Here, to expand through the periodic

(28) Kabalnov, A.; Wennerström, H. Langmuir 1996, 12, 276-292.
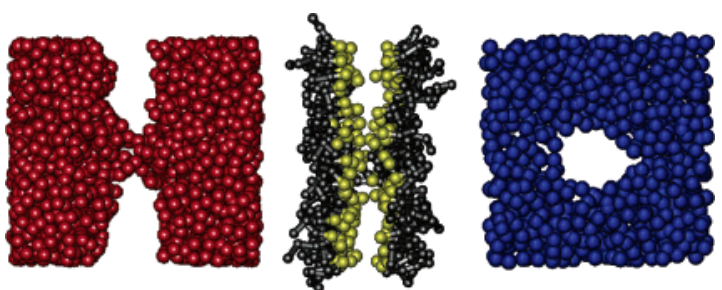

Figure 8. Snapshots from film rupture: (left) the two oil phases projected onto the $y-z$ plane; (middle) the surfactant monolayers projected onto the $y-z$ plane, headgroups are in yellow and tail groups in gray; (right) the water film projected onto the $x-y$ plane. The surfactant was of type ht $t_{4}$ and the density $N_{\text {surf }} / A$ $=0.6$.

boundary conditions, one of the two perpendicular oil cylinders must break off, to form one cylinder. This is a topological transition which costs energy and requires reorientation of molecules. It is an activated event just like the formation of the channel. After this second transition, a stable state with $S_{x}<0$ and $S_{y}=S_{z}>0$ is reached-this is identified as a cylinder parallel with the $x$ axis. The third transition is from a cylinder to a sphere where $S_{x}=S_{y}=S_{z}=0$. In Figure 7 the system finally fluctuates between a cylinder and a sphere. We identify the separation at which rupture occurs to be the separation at the first sharp drop in $S_{z}$. We stress that, although the subsequent transitions are due to the periodic boundary conditions, the channel formation is analogous to that between coalescing droplets.

Figure 8 shows snapshots of film rupture. The oil, surfactants, and water are depicted separately, but at the same time step-immediately after the water film ruptures, the surfactants bend to form a channel, and the two oil phases get in contact. Note that after the channel formation, fixing $N_{\text {surf }}$ in the simulation becomes a poor approximation since the local density of surfactant may change during droplet coalescence.

3.2. Droplet Separation. Interdroplet forces are typically presented as a function of droplet separation $h$, i.e., the thickness of the film separating the droplets. Here the interdroplet forces are measured at separations on the molecular scale. At this scale, the definition of interdroplet separation is not obvious.

The simulation box length corresponds to the separation between the plates in the surface force experiments. This distance is usually reported in experiments where the bior monolayers are supported on the rigid plates. Here there is sufficient bulk between the box walls and the interfaces to have free fluidlike interfaces. The separation between such interfaces is less trivial to define. ${ }^{9}$ Electrondensity distribution profiles can be used to locate the various components, although one would still have to make some choices. For example, McIntosh and Simon ${ }^{29}$ chose the inter-bilayer spacing as the distance between the headgroup peaks minus two headgroup radii. Density profiles can also be readily computed in the simulations. Figure 9 shows density profiles for two systems that are close to rupture. Figure 9a shows a water film between two oil phases, and Figure 9b shows an oil film between two water phases. In both cases, there are no molecules of the drop type in the middle, which means that the films did not yet rupture.

From Figure $9 \mathrm{a}$ it is possible to define an intermonolayer separation, based for example on the headgroup density peaks. The monolayers in Figure 9b, however, overlap. The inter-monolayer separation is thus an

(29) McIntosh, T. J.; Simon, S. A. Biochemistry 1986, 25, 40584066. 

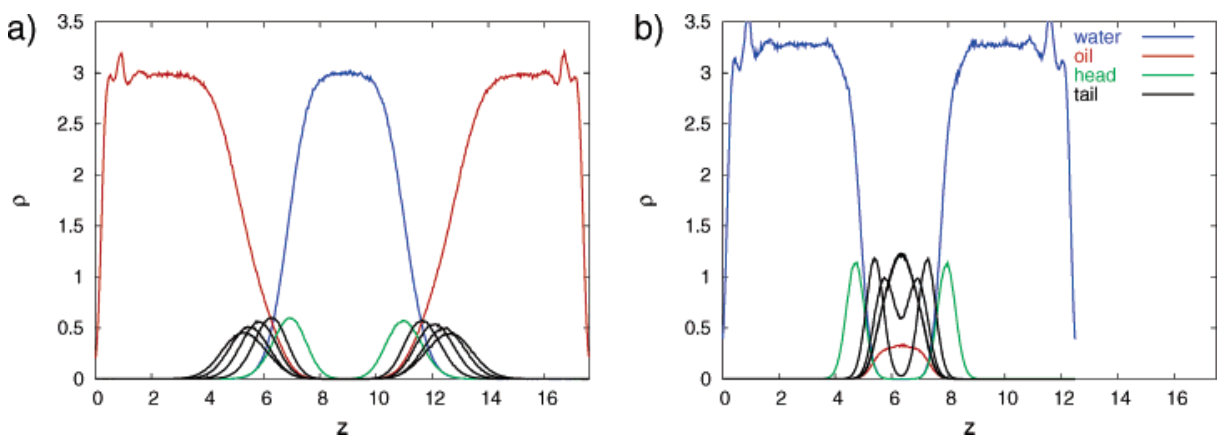

Figure 9. Density profiles at separations just before film rupture with ht ${ }_{4}$ surfactant and $N_{\text {surf }} / A=0.9$ : (a) two oil phases separated by a water film; (b) two water phases separated by an oil film.
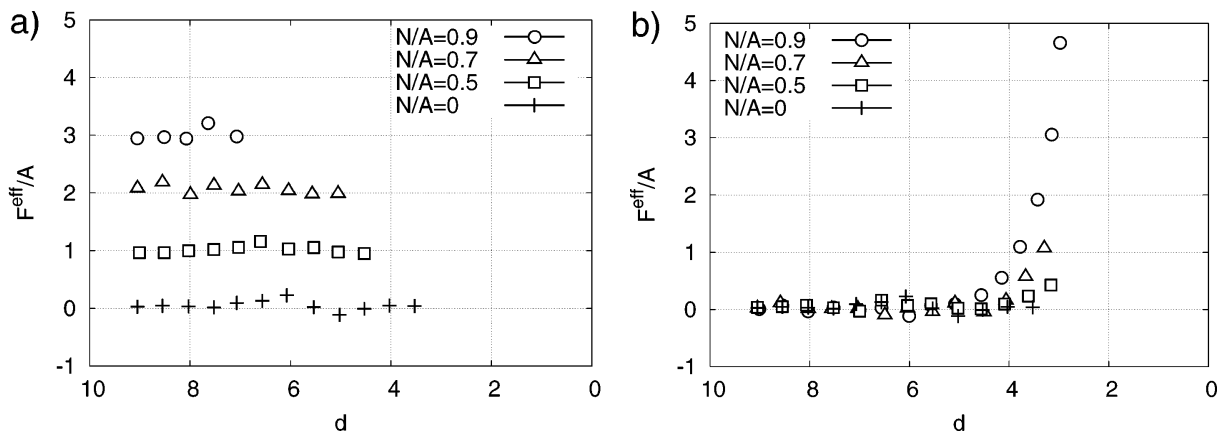

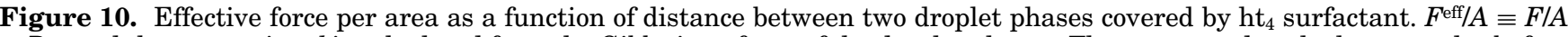
$-P^{\text {res }}$ and the separation $d$ is calculated from the Gibbs interfaces of the droplet phases. The curves end at the last samples before film rupture. N/A denotes the surfactant monolayer density. The four curves in (a) are essentially on top of each other but for clarity the curves for $N / A=0.5,0.7$, and 0.9 are shifted upward by 1,2 , and 3 units, respectively. (a) Two oil phases separated by a water film. (b) Two water phases separated by an oil film.

inconvenient parameter for the present study. We need a separation which is well defined up to the point when the film ruptures and the droplet contents mix.

In thermodynamics, phase boundaries are defined as the Gibbs dividing interface. The position of the Gibbs interface is determined on the basis of density profiles. We can in principle choose the separation as the distance between the Gibbs interfaces of the two drop phases (denoted $d^{\mathrm{d}}$ ) or of the film phase (denoted $d^{\mathrm{f}}$ ). From both graphs in Figure 9 we can define a Gibbs interface for each of the two drops and define the separation $d^{\mathrm{d}}$ as the distance between the two interfaces $s_{1}$ and $s_{2}$ :

$$
\begin{aligned}
& \int_{z_{1}}^{L_{z} / 2} \rho^{\mathrm{d}}(z) \mathrm{d} z=\rho_{\text {bulk }}^{\mathrm{d}}\left(s_{1}-z_{1}\right) \\
& \int_{L_{z} / 2}^{z_{2}} \rho^{\mathrm{d}}(z) \mathrm{d} z=\rho_{\text {bulk }}^{\mathrm{d}}\left(z_{2}-s_{2}\right)
\end{aligned}
$$

Here subscripts 1 and 2 refer to the two drop phases. $z_{1}$ and $z_{2}$ are arbitrary positions in the left and right bulk phases, respectively, taken between where the wall effects end and the interface starts. $\rho^{\mathrm{d}}(z)$ is the density of the component that the drops are made of, and $\rho^{\mathrm{d}}$ bulk is the density in the bulk of the droplet. Since the bulk oil and bulk water phases have identical properties, $\rho^{\mathrm{d}}$ bulk will differ from $\rho^{\text {res }}$ only at close separation when the pressure changes from that of the reference state.

In Figure 9a the film thickness $d^{\text {f }}$ can be calculated in a similar manner based on the density profile of the water film. In Figure 9b, however, the two surfactant monolayers are interdigitated and the oil molecules are mixed with the surfactant tails. Here it is difficult to talk about film thickness. Because there are only a few oil molecules left, $\rho_{\text {bulk }}^{f}$ is not defined. However, the number of film molecules divided by area is well defined and we chose $N_{\text {film }} / A$ as an alternative characterization of film thickness. The ad- vantage is that $N_{\text {film }} / A$ is well defined also in the nonequilibrium simulations. Here, density profiles cannot be computed because the box length changes continuously. However, for the equilibrium simulations, $d^{\mathrm{d}}$, based on the Gibbs interface of the droplet phase, is the best choice (in the following sections $d^{\mathrm{d}}$ will be noted as $d$ ).

\section{Results and Discussion}

4.1. Surface Forces in Oil-in-Water and Water-inOil Emulsions. Figure 10 shows force-distance curves for oil droplets separated by a water film (a) and water droplets separated by an oil film (b), with and without surfactants. The surfactants are of type $\mathrm{ht}_{4}$. The case of zero surfactants is shown in both plots and is identical due to the oil-water symmetry of the model. In parts a and $b$ of Figure 10 the monolayers are facing each other heads-on and tails-on, respectively. The curves are from equilibrium simulations and end at the shortest separation at which the film did not rupture. The figures show a clear difference between oil films and water films. The water films break without a barrier, whereas the oil films break with a barrier that increases with the density of surfactants at the interface.

Several types of forces may act between two dispersed droplets: Classical colloid science features the DLVO forces, which include van der Waals and electrostatic interactions, but a number of other interactions are known to play a role in emulsion stability: depletion forces due to soluble polymers or micelles in the film, oscillatory structural forces due to layering of these, and steric forces due to overlap between chains adsorbed at opposite droplets. ${ }^{30}$ Entropic forces due to the thermal density

(30) Petsev, D. N.; Denkov, N. D.; Kralchevsky, P. A. J. Colloid Interface Sci. 1995, 176, 201-213. 

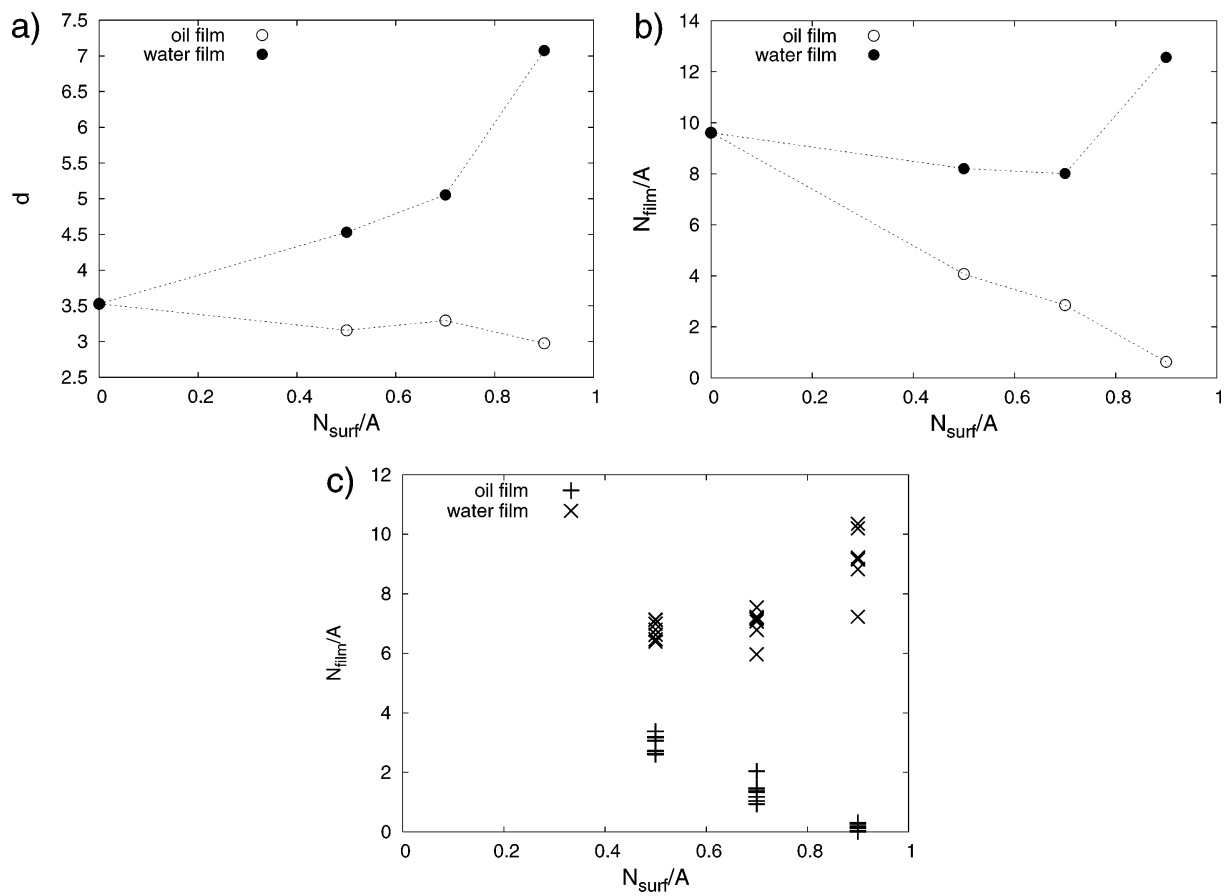

Figure 11. (a) Separation just before film rupture as a function of surfactant density at the interface sampled in equilibrium simulations. The surfactant was of type $\mathrm{ht}_{4}$. The dotted lines are guides to the eye. (b) Number of film molecules per unit area ("film thickness") just before film rupture as a function of surfactant density at the interface sampled in equilibrium simulations. The dotted lines are guides to the eye. (c) Number of film molecules per unit area ("film thickness") at film rupture as a function of surfactant density at the interface sampled in nonequilibrium simulations. The compression speed was $\left(2 \times 10^{-5}\right) r_{\mathrm{c}}$ per MC cycle.

fluctuation of liquid interfaces include undulation and protrusion forces. ${ }^{31}$

DLVO forces are not expected for the simple force models studied here. Furthermore, oscillatory effects due to solvent packing vanish for liquid interfaces. ${ }^{9}$ The repulsive forces in Figure 10b arise at the separation where the tails start to interdigitate. The force is thus steric and of the same type as those observed with polymer brushes in good solvents. ${ }^{11}$

Of the forces listed above, only steric and undulation forces would be expected for this model. Undulation forces are entropic repulsive forces due to the thermal undulations of the monolayers. Previously we characterized these undulations in terms of the bending moduli. ${ }^{5,6}$ However, the present $L_{x}$ and $L_{y}$ are only one to two times the lowest undulation wavelength $\lambda$ that follows the predicted $\lambda^{-4}$ spectrum. This suggests that most undulation modes are suppressed by the limited system size. Furthermore, although such an undulation force would be of a repulsive nature, it is possible that the undulatory fluctuations actually induce channel formation by perturbing locally the monolayers' curvature and density. If this is the case, i.e., if the interface ruptures at a separation larger than the range of the undulatory forces, they would not be observed within the present simulation system.

4.2. Separation at Film Rupture. The force curves shown in the previous section are sampled in equilibrium simulations at intervals of $0.5 r_{\mathrm{c}}$. Data are shown only for separations where the film did not rupture during the simulation. The separation at rupture depends to some extent on the sampling time, i.e., rupture could sometimes occur at a separation larger by 0.5 or 1 unit if we sampled longer. However, all simulations were equally long and we can therefore study the qualitative effect of surfactants by comparing the rupture separations. Figure 11a shows

(31) Israelachvili, J. N.; Wennerström, H. J. Phys. Chem. 1992, 96 , 520-531. the separation in the last sample before rupture took place. For water films, i.e., for two monolayers with headgroups facing each other, we observe that rupture occurs at a larger separation when the surfactant density increases. Comparing two pairs of approaching oil droplets, this means that the pair with the higher surfactant density will coalesce first. For water droplets separated by an oil film, the separation at coalescence seems to be independent of the surfactant density.

Figure 11b shows the number of film molecules per unit area, rather than the Gibbs distance between the droplet phases. This property can be thought of as a qualitative measure of the film thickness. The conclusions differ slightly from those drawn on the basis of Figure 11a: The oil film thickness at rupture decreases dramatically with increasing surfactant density. Conversely, the water film thickness at rupture does not depend strongly on the thickness.

Since the thickness of the surfactant monolayer is of the order of the film thickness, the quantitative dependence on surfactant density depends on whether the film thickness or the droplet separation is used as reference. The graphs show that the water films rupture when the number of water particles within a certain area is roughly the same, independent of the surfactant density. As there are more surfactants between the droplets and the film when the surfactant density increases, this implies that the droplet distance at coalescence increases more rapidly with density. For the same reason, the pronounced decrease in amount of oil between two coalescing water droplets must be accompanied by a lesser decrease in droplet distance. This explains the difference between parts $a$ and $b$ in Figure 11. These results are clearly physical. The apparent ambiguities arise because we investigate at the molecular scale. Nevertheless, both graphs indicate that an increasing density of $\mathrm{Ht}_{4}$ surfactant has a positive effect on the stability of water droplets and a negative effect on the stability of oil droplets. 


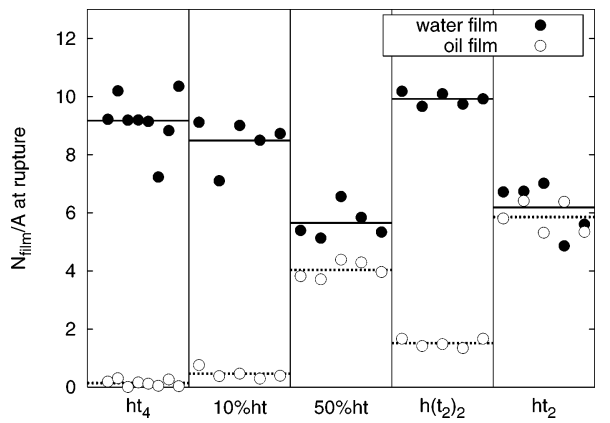

Figure 12. Film thickness $N_{\text {film }} / A$ at rupture sampled in nonequilibrium simulations for various surfactant structures. $L_{z}$ was reduced by $\left(2 \times 10^{-5}\right) r_{\mathrm{c}}$ per MC cycle. Results of five independent simulations are shown (eight for $\mathrm{ht}_{4}$ ), and the horizontal bars denote the average. $10 \%$ ht means a mixture of $90 \% \mathrm{ht}_{4}$ and $10 \% \mathrm{ht}$. The surfactant density is $N_{\text {surf }} / A=0.9$.

To improve the resolution and check the reproducibility of the results, we performed nonequilibrium simulations, in which the box length was decreased continuously as described in section 2.5. The Gibbs separation $d$ could not be obtained from these nonequilibrium simulations, as density profiles are somewhat ill-defined for a continuously decreasing box length. Instead, we plotted the instantaneous $N_{\text {film }} / A$ (Figure 11c). Eight parallel runs were performed for each case. The reproducibility is quite good, and the main trends are consistent with parts $a$ and $b$ of Figure 11. In essence, with ht $_{4}$ surfactants, oil-wateroil films break earlier and water-oil-water films break later when the surfactant density increases.

Let us relate these results to experimental and theoretical knowledge about emulsion stability. Bancroft's rule states that oil-soluble surfactants tend to stabilize waterin-oil emulsions, and water-soluble surfactants tend to stabilize oil-in-water emulsions. The $\mathrm{ht}_{4}$ surfactant is more soluble in oil than water, because it has more lipophilic than hydrophilic beads. Therefore, the conclusions drawn from Figure 11 agree with Bancroft's rule.

The system considered here resembles creamed or sedimented layers of closely packed droplets, where the coalescence rate depends on a thermally activated film rupture. This case was considered by Kabalnov and Wennerström. ${ }^{28}$ They developed a channel nucleation theory based on the energy penalty of forming a channel between the two droplet phases. The channel free energy depends strongly on the bending modulus $\kappa$ and the spontaneous curvature $c_{0}$ of the surfactant monolayer. The theory provides a molecular basis for emulsion stability: For surfactants with a negative spontaneous curvature (i.e., preferring to bend toward the water phase), creating a channel between two oil-water-oil films implies, on average, bending the monolayers toward the spontaneous curvature. Conversely, creating a channel between two water-oil-water films involves bending against the spontaneous curvature. The observation from Figure 11 is that with $\mathrm{ht}_{4}$ surfactant, molecular fluctuations lead to channel formation easier or earlier in oilwater-oil films than in water-oil-water films. Since the $\mathrm{ht}_{4}$ surfactants have a small hydrophilic group and a longer, thermally vibrating tail, it is reasonable to assume that their spontaneous curvature is negative. Our findings are thus in agreement with the predictions from the channel nucleation theory in ref 28 . We have estimated ${ }^{6}$ $\kappa$ to increase from $1 k_{\mathrm{B}} T$ to $2 k_{\mathrm{B}} T$ in the density range $N_{\text {surf }} / A$ $=0.5-0.9$. In the future we plan to calculate the free energy barrier using appropriate biased simulation techniques.

4.3. Effect of Surfactant Structure on the Rupture Separation. Figure 12 shows the box length at rupture for various surfactants at a monolayer density of $N_{\text {surf }} / A$ $=0.9$. We compared the linear $\mathrm{ht}_{4}$ surfactants with mixtures of $\mathrm{ht}_{4}$ and $10 \%$ and $50 \%$ cosurfactants (ht), its branched isomer $\mathrm{h}\left(\mathrm{t}_{2}\right)_{2}$, and the shorter surfactant $h t_{2}$.
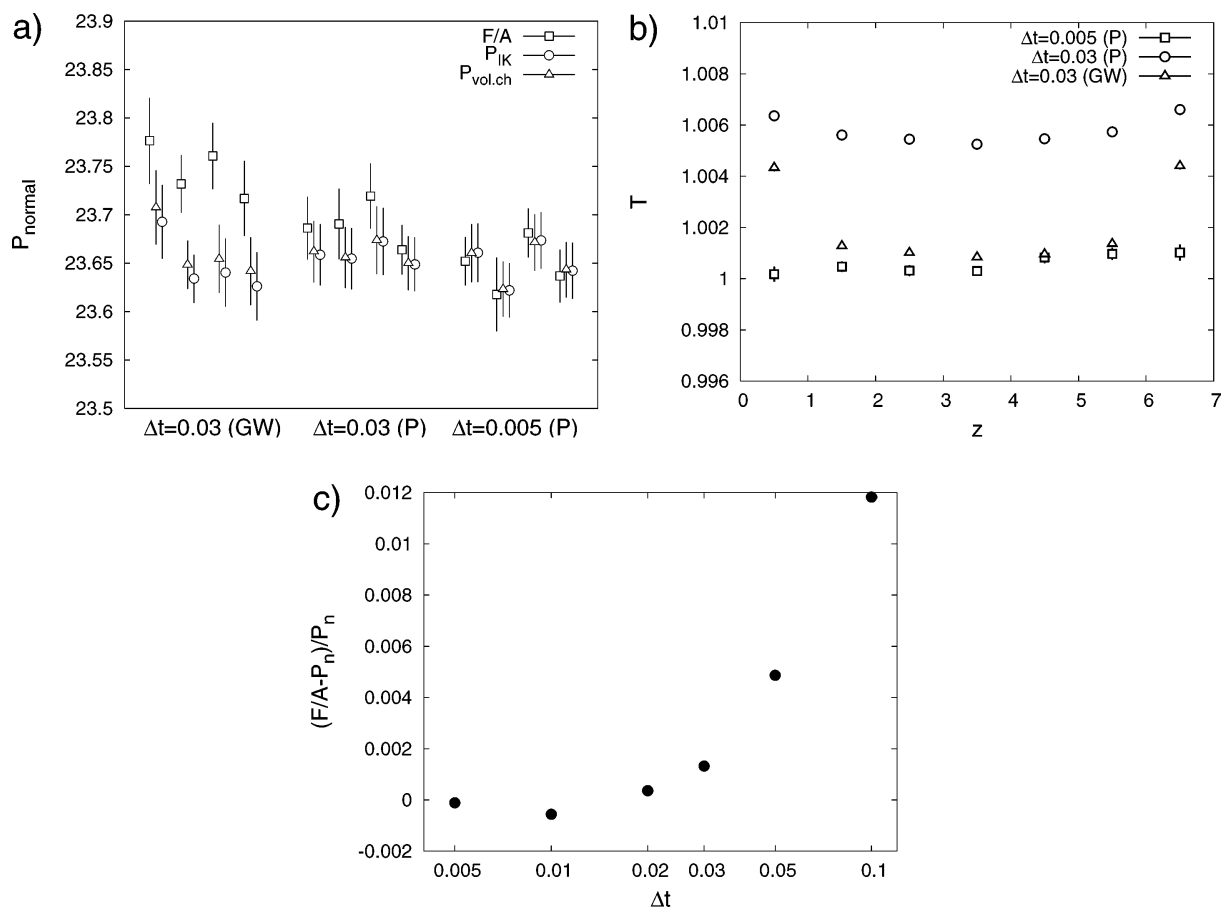

Figure 13. (a) The pressure in direction normal to the walls calculated in three different ways. For $\Delta t=0.03, F / A>P_{\text {vol.ch }}>P_{\text {IK }}$ The four left simulations $(\mathrm{GW})$ were obtained using the Groot Warren integration scheme, the remaining $(\mathrm{P})$ were obtained with the Peters thermostat. (b) The temperature profile in the box. The increase toward the walls is due to the finite time step and explains the order in (a). (c) The difference between wall forces and bulk pressure as a function of time step with the Peters thermostat. 
The difference between water films and oil films decreases upon addition of cosurfactant. Cosurfactants cause film rupture to occur at a larger separation for oil films and a smaller separation for water films compared to pure $\mathrm{ht}_{4}$. This is also in agreement with the channel nucleation theory since cosurfactants reduce the absolute value of $c_{0}$, making it less negative in this case. Branching of the tail, on the other hand, reduces $c_{0}$ and is expected to shift the stability toward water-in-oil emulsions. However, Figure 12 shows that branching decreases the stability of both the oil and water films. Finally, $\mathrm{ht}_{2}$ is a nearly balanced surfactant with $c_{0} \approx 0$. There is therefore an insignificant difference between the oil and water films, and the results are also similar to those for a 50 mixture of $\mathrm{ht}_{4}$ and ht.

\section{Conclusion}

We have presented a system to study surface forces and film rupture with molecular simulations. We studied nonionic model surfactants for which the major contribution to the surface forces stem from steric chain-chain interactions. We found that a water film separating two surfactant-coated oil droplets breaks spontaneously when the oil droplets approach each other. When the monolayer density increases, the separation at which film rupture occurs increases. Conversely, an external force must be applied to overcome the steric repulsion between two water droplets coated with the same surfactant. Rupture could be induced only by forcing the interfaces together and the separation at rupture is less sensitive to the surfactant density. These surfactants will therefore have a positive effect on the stability of water-in-oil emulsions and a negative effect on the stability of oil-in-water emulsions.

A. Effect of the Integration Scheme on System Equilibrium. In mechanical equilibrium the force per area on each of the walls equals the normal pressure in the system. As a consistency check these forces were computed in three independent ways.

(1) The force on the walls is a sum of the soft repulsive forces exerted by the particles and the change in impulse due to the hard reflective walls

$$
F^{\text {wall,left }}=\sum_{i} F_{i}^{\text {wall,left }}+\sum_{j, \text { reflected }} \frac{m \Delta v_{j}}{\Delta t}
$$

(2) The system was divided into 500 slabs in the $z$ direction. In the slabs positioned with $r_{\mathrm{c}}<z_{\text {slab }}<L_{z}-r_{\mathrm{c}}$, the pressure tensor has no contribution from the walls. It can then be calculated using the Irving-Kirkwood method. ${ }^{32}$ By averaging the normal component over these slabs, we obtain an average in this part of the system, denoted $P_{\mathrm{IK}}$.

(3) A third way of calculating the normal pressure is the trial volume change method. ${ }^{33}$ The box length is virtually rescaled from $L_{z}$ to $L_{z}+\Delta z$ where $\Delta z$ is a random number between -0.05 and 0.05 . All particle positions are rescaled from $z_{\mathrm{i}}$ to $z_{\mathrm{i}}+\Delta z z_{\mathrm{i}} / L_{z}$, and the change in potential energy $\Delta U$ is computed. The normal pressure is then

$$
P_{\text {vol.ch }}=\frac{N}{V} k_{\mathrm{B}} T-\frac{\Delta U}{L_{x} L_{y} \Delta z}
$$

With $\Delta t=0.03$ and the integration scheme of Groot and Warren, ${ }^{17}$ which we used in previous studies of these systems, there is a systematic difference between these three methods with $F / A>P_{\text {vol.ch }}>P_{\text {IK. }}$ This is shown in Figure 13a. The difference disappears by removing the random and dissipative forces and reducing the time step to 0.005 (molecular dynamics). The difference was also reduced significantly by using the Peters thermostat ${ }^{20}$ without reducing the time step. The discrepancy between the three methods of calculating the pressure must arise from a lack of equilibrium in the system. We divided the system into slabs of thickness $r_{\mathrm{c}}$ and assigned to the slab a temperature

$$
T_{\text {slab }}=\frac{N}{(N-1)} \frac{1}{3 N_{\text {slab }}} \sum_{i \text {-in-slab }} m v_{i}^{2}
$$

where the sum is over all particles in the slab. Figure $13 \mathrm{~b}$ shows that the temperature close to the walls is higher than that in the bulk for both schemes at $\Delta t=0.03$. Since $F / A$ is sampled only close to the walls, $P_{\mathrm{IK}}$ is sampled only away from the walls, and $P_{\text {vol.ch }}$ in the whole system, this explains why $F / A>P_{\text {vol.ch }}>P_{\text {IK. }}$. With the Peters thermostat, the temperature gradients and pressure discrepancies can be removed by decreasing the time step (Figure 13c). However, with this thermostat the discrepancy is within an acceptable error even for $\Delta t=0.03$. Figure $13 \mathrm{~b}$ show that at $\Delta t=0.03$, the global temperature in the system deviates more from the thermostat set point for the Peters thermostat than the Groot and Warren scheme. However, the temperature is more uniform with the Peters thermostat. This can be explained as follows: Integrating the equation of motions in DPD with a finite time step is a nontrivial task as the velocity-dependent forces disable straightforward use of the Verlet scheme commonly used in molecular dynamics simulations. An early effort was made by Groot and Warren ${ }^{17}$ who found a scheme which, with a certain combination of parameters, minimized the temperature deviation at $\Delta t=0.03$. The excellent temperature conservation is unfortunately due to two errors that cancel at this time step. ${ }^{34}$ The inaccurate integration of the dissipative force cancels the error in integration of the conservative force that stems from the large time step. Although this gives good temperature control, it is uncertain what other effects this cancellation of errors have on other properties of the system. One such unfortunate effect is the temperature gradient in Figure $13 \mathrm{~b}$. The DPD algorithm recently proposed by Peters ${ }^{20}$ works differently. Rather than integrating the dissipative and random forces, relative particle velocities are thermostated after the integration of the conservative forces that can be done with the Verlet scheme. The Peters thermostat rigorously maintains the Maxwell distribution of the velocities for any time step. Thus, any deviation in temperature from the thermostat set point is solely due to the finite time step used to integrate the conservative forces. This could also be achieved with the Anderson or Lowe thermostats; ${ }^{19}$ the advantage with the Peters thermostat is that it reduces to the original dissipative and random forces in the limit of $\Delta t \rightarrow 0$. On the basis of this and the results shown in Figure 13, we chose to proceed with the Peters thermostat. Although the Groot and Warren scheme gives a global temperature closer to the

(32) Irving J. H · Kirkwood, J G. J Chem Phys. 1950, 18, 817-829.

(33) Frenkel, D.; Smit, B. Understanding Molecular Simulations: from Algorithms to Applications, 2nd ed.; Academic Press: San Diego, CA, 2002 .

(34) Hafskjold, B.; Liew, C. C.; Shinoda, W. Mol. Simul., in press. 
thermostat set point, it is more important to have equilibrium in the system. A smooth temperature profile at $T=1$ could only be achieved by decreasing the time step, which requires more computer time. Figure $13 \mathrm{c}$ shows the difference between the wall forces and bulk pressure as a function of time step with the Peters thermostat. We chose to stick with a time step $\Delta t=0.03$ and accept an error of $0.2 \%$ in the wall forces. We reported $F / A$ in the results section since its computation requires no extra CPU time.

We also tried wall potentials that were repulsive enough to prevent particles from crossing the walls even without the hard reflection (eqs 10 and 11), e.g.

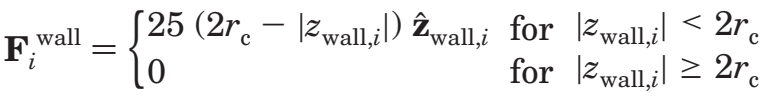

This did not affect the difference between the wall forces and the pressure away from the forces.

Acknowledgment. These investigations are supported in part by the Norwegian Research Council (Grant No. 145184/432), by The Netherlands Research Council for Chemical Sciences (CW), and by The Netherlands Organization for Scientific Research (NWO) through PIONIER.

LA048071P 